\title{
Findings from post-intervention analysis of pre-eclampsia/ eclampsia in Cross River State
}

Salisu Mohammed Ishaku

Population Council

Follow this and additional works at: https://knowledgecommons.popcouncil.org/departments_sbsr-rh

Part of the Demography, Population, and Ecology Commons, Family, Life Course, and Society Commons, International Public Health Commons, Maternal and Child Health Commons, and the Women's Health Commons How does access to this work benefit you? Let us know!

\section{Recommended Citation}

Ishaku, Salisu Mohammed. 2018. "Findings from post-intervention analysis of pre-eclampsia/eclampsia in Cross River State," Ending Eclampsia Research Brief. Abuja: Population Council. 
Findings from post-

intervention analysis

of pre-eclampsia/

eclampsia in

Cross River State
The Population Council conducts research and delivers solutions that improve lives around the world. Big ideas supported by evidence: It's our model for global change. popcouncil.org

(c) 2018 The Population Council, Inc.


FROM THE AMERICAN PEOPLE

Made possible by the generous support of the American people through the United States Agency for International Development (USAID).

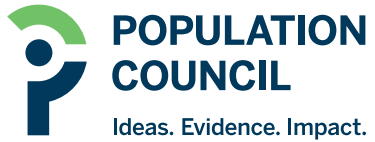




\section{BACKGROUND}

Despite global efforts to reduce preventable maternal and neonatal mortality, Nigeria's maternal mortality ratio is estimated at 576 deaths per 100,000 live births and neonatal death is estimated at 37 per 1,000 live births ${ }^{1}$.

Maternal and newborn deaths due to pre-eclampsia and eclampsia (PE/E) are preventable, yet in Nigeria this is the most significant direct cause of maternal mortality.

Following a landscape analysis to better understand the enormity of this problem across seven states in Nigeria, a cross-cutting intervention was implemented in Cross River, Ebonyi, and Kogi states. Researchers worked with primary health care (PHC) providers, policy makers, women's groups, and community members to increase uptake of underutilized interventions and commodities for the prevention and treatment of PE/E in rural Nigeria. Followup analysis occurred in each state. This brief presents study findings from Cross River State on post-intervention landscape changes in:

- Programmatic and policy support for PE/E prevention and treatment;

- PHC facilities' capacity to manage PE/E;

- Provider knowledge and competence in preventing, detecting, and managing PE/E; and

- Community awareness, beliefs, and experiences around PE/E.

\section{METHODS}

This PE/E study is a pre/post, cross-sectional design with qualitative and quantitative data collected at baseline (2015) and endline (2018). Data collection activities include in-depth interviews (IDIs) with policy makers, survivors of PE/E and laboratory professionals; focus group discussions (FDGs) with men and women from the community; and surveys and observations to assess provider capacity in antenatal care and facility readiness and capacity to identify, treat, and manage PE/E at the primary and secondary levels. Table 1 describes data collection activities at baseline and endline in Cross River State.

\begin{tabular}{|l|c|c|}
\hline \multirow{2}{*}{ Table 1: Data collection activities in Cross River } \\
\cline { 2 - 3 } & Baseline & Endline \\
\hline IDIs with policy makers & 10 & 18 \\
IDIs with PE/E survivors & 5 & 6 \\
IDIs with laboratory professionals & 4 & 6 \\
FGDs with married men \& women & 4 & 4 \\
Surveys with providers & 54 & 74 \\
Assessments of health facilities & 11 & 54 \\
Observations of ANC visits & 15 & 240 \\
\hline
\end{tabular}

PRE-ECLAMPSIA/ECLAMPSIA

(PE/E) IN BRIEF

- $\mathrm{PE}$ is a condition in pregnant women marked by an increase in blood pressure and protein in urine after 20 weeks gestation; eclampsia is a life-threatening condition characterized by convulsions in women with PE.

- Providing high quality ANC improves the prevention and early detection of $\mathrm{PE}$ and can prevent its progression to eclampsia.

- $\mathrm{PE} / \mathrm{E}$ can be managed by administering anti-hypertensive drugs and $\mathrm{MgSO}_{4}$.

\section{CORE INTERVENTION}

- Intervention research occurred in Cross River, Ebonyi, and Kogi states, in 2016 and 2017, and follow-up data was collected from May-June 2018.

- 557 primary health care (PHC) providers received one month of training with continuous mentorship to identify hypertension and PE/E, treat with $\mathrm{MgSO}_{4}$, and refer to secondary facilities.

- The primary objective was to facilitate task-shifting to $\mathrm{PHC}$ providers of $\mathrm{MgSO}_{4}$ loading dose administration and secondary facility referral for improved PE/E detection, treatment, and management.

\section{ADDITIONAL WOMEN'S GROUP INTERVENTION}

- 46 women leaders in 24 communities received training to deliver health education regarding ANC and PNC to women's groups in their communities.

- 28 women leaders from intervention and comparison groups and 12 women's group attendees were interviewed post-intervention. 


\section{POLICY INFLUENCE}

Researchers interviewed 18 policy makers from the local and state levels to understand their knowledge of programs and policies to detect, prevent, and manage $\mathrm{PE} / \mathrm{E}$ in Cross River State. Policymakers were asked about efforts in their LGA or state to advance national PE/E policies; their support for task shifting to $\mathrm{PHC}$ providers for $\mathrm{PE} / \mathrm{E}$ management in accordance with national protocol; and about the utilization of antihypertensive drugs and magnesium sulfate $\left(\mathrm{MgSO}_{4}\right)$ in their communities.

State policy makers in Cross River State know that a national policy exists in Nigeria regarding management of $\mathrm{PE} / \mathrm{E}$, however not all could articulate the contents of this protocol. Contrastingly, few policy makers or health managers at the LGA level knew of or could describe the national protocol. State policy makers indicated that copies of the national protocol could be found at the Federal Ministry of Health.

Encouragingly, state policy makers and LGA health managers support task shifting to PHC workers to provide a loading dose of $\mathrm{MgSO}_{4}$ and refer women to secondary facilities.

Policy makers emphasize the importance of adequate and appropriate training, and there is generalized consensus that with sufficient training, PHC workers can reduce preventable deaths for women with severe preeclampsia (SPE) or eclampsia.

\section{"Task shifting is a welcome development because we only have a few nurses in the LGAs, so the task should be shifted from nurses to other health care workers so that if the nurse is not there at the community level to administer, it doesn't mean the patient should die."}

- State policy maker, 2018

\section{FACILITY CAPACITY \& PREPAREDNESS}

At endline, researchers visited 54 facilities in Cross River State and compared reports from facility managers in 11 facilities where data was collected prior to the intervention.

At baseline, only five (45\%) facilities had all the necessary ANC equipment to detect PE/E. At endline, 45 facilities (83\%) had all equipment. At baseline, $46 \%$ of facilities used $\mathrm{MgSO}_{4}$ to manage $\mathrm{PE} / \mathrm{E}$, but at endline, 33 facilities (61\%) reported using $\mathrm{MgSO}_{4}$ to manage $\mathrm{PE} / \mathrm{E}$.

Through direct observation, researchers identified the number of facilities that stocked key equipment to detect and manage $\mathrm{PE} / \mathrm{E}$ and treat $\mathrm{MgSO}_{4}$ toxicity. Improvements were observed for stocking all key equipment except urine bags, though the overall number of facilities with urine bags increased 6\% (Figure 1). There was an increase in the number of $\mathrm{PHC}$ facilities that used $\mathrm{MgSO}_{4}$ to treat $\mathrm{PE} / \mathrm{E}$ at endline compared to baseline.

Though not statistically significant, more facility managers reported sometimes using $\mathrm{MgSO}_{4}$ (14/54 vs. 1/11 at baseline). Fewer than half of managers at endline said $\mathrm{MgSO}_{4}$ is never used in their facilities $(p=0.01)$. These findings suggest that, while gaps persist, more facilities can procure $\mathrm{MgSO}_{4}$ for patients when needed.

Whereas at baseline, $80 \%$ of the facilities that stocked $\mathrm{MgSO}_{4}$ received it as part of their regular supply from central medical stores, an equal number of facilities receive it as part of the central supply or purchase it for patients from a local pharmacy. At baseline and endline, only $20 \%$ of facilities in Cross River State require patients to purchase $\mathrm{MgSO}_{4}$ themselves.

A critical component of managing and treating severe $\mathrm{PE} / \mathrm{E}$ is the capacity to conduct emergency obstetric and neonatal care (EmONC) by providing key signal functions

Figure 1: Facilities with key equipment to detect and manage $\mathrm{PE} / \mathrm{E}$ and $\mathrm{MgSO}_{4}$ toxicity (\%)

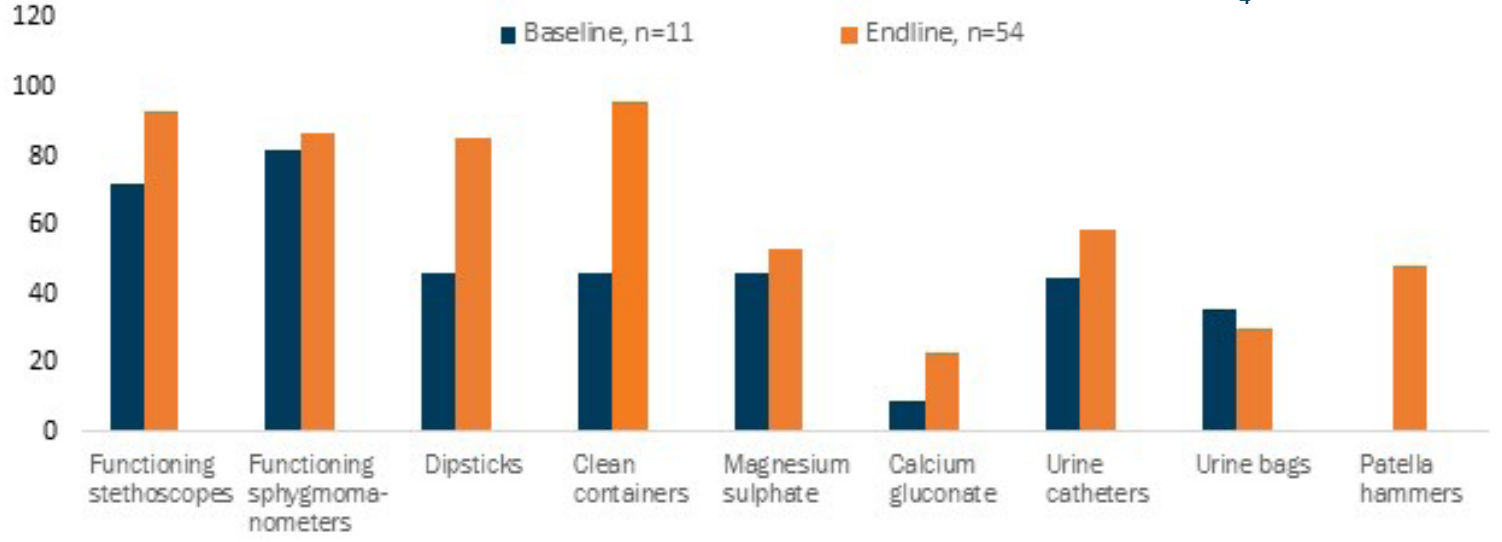


and essential drugs to mothers throughout the pregnancy continuum (pregnancy, labor and delivery, and postnatal periods).

In Cross River State, eight facilities (15\%) stock all essential equipment, and 27 (50\%) stock at least half of the essential supplies. Table 2 shows facilities' capacity to provide EmONC signal functions and records of having done so in the last three months.

\begin{tabular}{|c|c|c|c|c|}
\hline & \multicolumn{2}{|c|}{ Baseline, $n=11$} & \multicolumn{2}{|c|}{ Endline, $n=54$} \\
\hline & Capacity & $\begin{array}{l}\text { Done } \\
\text { in past } \\
3 \text { mos }\end{array}$ & Capacity & $\begin{array}{c}\text { Done } \\
\text { in past } \\
3 \text { mos }\end{array}$ \\
\hline $\begin{array}{l}\text { Perenteral } \\
\text { antibiotics }\end{array}$ & 6 & 6 & 39 & 32 \\
\hline Parenteral oxytocics & 8 & 8 & 43 & 39 \\
\hline $\begin{array}{l}\text { Parenteral } \\
\text { anticonvulsants } \\
\left(\mathrm{MgSO}_{4}\right)\end{array}$ & 4 & 4 & 27 & 14 \\
\hline $\begin{array}{l}\text { Manual placenta } \\
\text { removal }\end{array}$ & 11 & 5 & 40 & 25 \\
\hline $\begin{array}{l}\text { Retained products } \\
\text { removal }\end{array}$ & 8 & 6 & 33 & 19 \\
\hline $\begin{array}{l}\text { Assisted vaginal } \\
\text { delivery }\end{array}$ & 7 & 4 & 33 & 28 \\
\hline Blood transfusion & 4 & 4 & 16 & 13 \\
\hline Caesarean section & 3 & 4 & 10 & 9 \\
\hline $\begin{array}{l}\text { Neonatal } \\
\text { rescuscitation }\end{array}$ & 7 & 4 & 42 & 32 \\
\hline
\end{tabular}

\section{PROVIDER KNOWLEDGE \& SKILLS}

Seventy-four providers from primary and secondary facilities in Cross River State responded to questions about preventing, detecting, and treating $\mathrm{PE} / \mathrm{E}$. At endline, there was a 12 percentage-point increase in the number of providers able to correctly identify preeclampsia (from $69 \%$ at baseline to $81 \%$ at endline), though this change was not statistically significant. Rates of providers' correct identification of hypertension in pregnancy remained high at approximately $94 \%$.

A key component of task shifting from secondary to primary facilities is PHC providers' capacity to prevent and manage $\mathrm{PE} / \mathrm{E}$ using antihypertensive drugs, $\mathrm{MgSO}_{4}$, and calcium gluconate to treat $\mathrm{MgSO}_{4}$ toxicity. Figure 2 highlights knowledge improvements among PHC providers from baseline to endline.

This intervention is based on the Pritchard method for administering loading and maintenance doses of $\mathrm{MgSO}_{4}$ to treat severe PE and prevent the onset of eclampsia. While only $10 \%$ of providers could correctly describe the loading and maintenance doses of $\mathrm{MgSO}_{4}$
Figure 2: Providers' knowledge of drugs for PE/E prevention and management (\%)

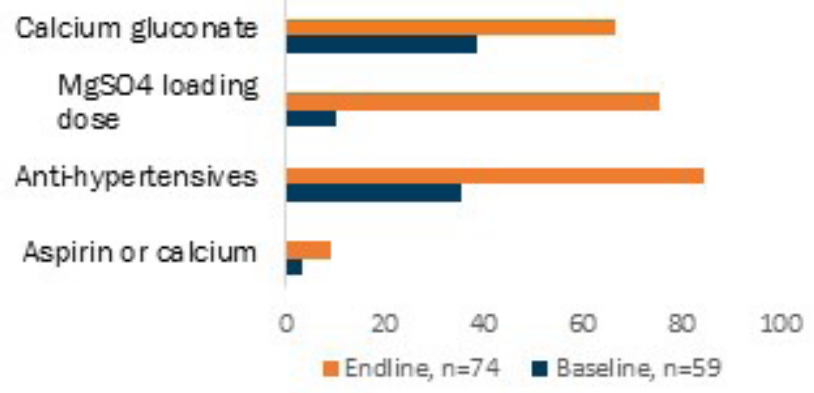

at baseline, significantly more (58\%) now know the correct maintenance dose and more than three-quarters (76\%) know the appropriate loading dose $(p<0.001$ and $p<0.001$, respectively). Similarly, whereas at baseline less than half of providers knew the correct antidote to $\mathrm{MgSO}_{4}$ toxicity, at endline more than two-thirds of providers recognize calcium gluconate as the correct antidote $(p=0.002)$.

\section{QUALITY OF CARE}

Quality of care was assessed through 240 observations of client-provider interactions during ANC visits, as well as qualitative data generated through four community focus groups with married men and women and six interviews with $\mathrm{PE} / \mathrm{E}$ survivors. Figure 3 compares the proportions of ANC components that clients received at baseline and endline.

Changes in client-provider interactions are mixed with several important improvements, as well as, several decreases in services. As at baseline, virtually all clients had their blood pressure (BP) and weight checked during the observed ANC visits, but fewer women received tests for proteinuria. Interviews with laboratory staff reveal bottlenecks to supply procurement, most often tests for urinalysis, that result from financial challenges at the government level.

\section{"We try in most cases not to experience \\ [shortages of urinalysis supplies], but because sometimes you request for funds to be able to buy and it is not approved, we sometimes run out of stock."}

-Chief laboratory scientist, general hospital, 2018

Qualitative reports from PE survivors indicate that, in addition to screening them for high BP and proteinuria, providers at endline took time to read the women their test results. Women report learning about PE/E from their providers, though education is frequently on potential consequences rather than warning signs of $\mathrm{PE} / \mathrm{E}$ or health behavior improvements. 


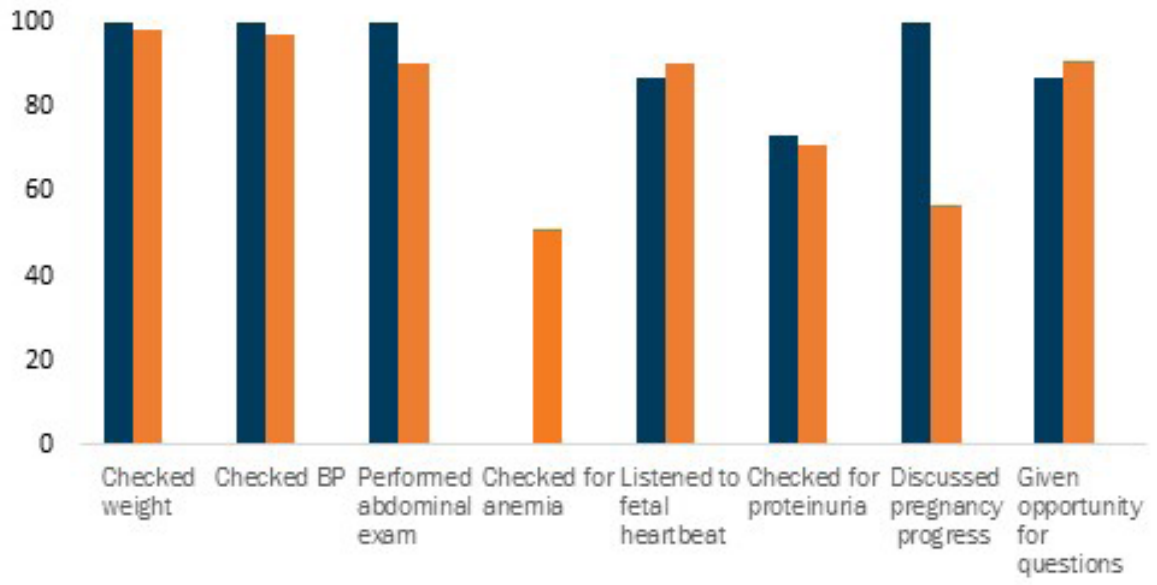

"[The provider] will ask me to go for urine test each time and they will check one other thing. He will tell you that he doesn't want pre-eclampsia to occur that is why he's begging all the pregnant women to register in the clinic. Because when you come anytime you have your date for check-up they will run those two tests."

-Pre-eclampsia survivor, 2018

Female focus group participants brought up a woman's fear of provider mistreatment as a potential barrier to women's health seeking behaviors; however, no personal experiences were mentioned. Many women, both survivors and focus group participants, reported frequent ANC attendance and deliveries at health facilities.

\section{"Even though they declare free treatment, not every women will deliver at the hospital. Some people like to deliver at the hospital; some people like to deliver at home because some women fear that if they go to the hospital they will be beaten... it is fear."}

- Female FGD participant, 2018

\section{COMMUNITY KNOWLEDGE \& PERCEPTIONS}

In contrast to baseline findings, traditional remedies for hypertension-related complications in pregnancy, such as the use of palm oil and herbs, appear much less prevalent in Cross River state. Men and women, when asked to describe how communities and individuals deal with complications during pregnancy, recommended visiting the hospital for appropriate care.

Some women still choose to seek care from religious leaders and through prayer, but religious leaders now actively participate in the health system and are a referral mechanism for women needing health services. Among the groups surveyed, myths and mistrust between communities and health providers appear to be less of a barrier to health facility use. While traditional medicine and religious influences are still prevalent, the importance of seeking health care services at primary or secondary facilities is well-understood among community members, including women, their husbands, and mothers-in-law.

\section{"ANC is important because the woman knows that attacks on her will be much. To dodge any attack, to believe she is free from it, those check-ups are one way that the attacks won't come."}

\section{- Male FGD participant, 2018}

Compared to baseline data, bewitching or spiritual attacks rarely surfaced as reasons for pregnancyrelated complications during community discussions; however, many signs and symptoms of $\mathrm{PE} / \mathrm{E}$ are still attributed to a woman's over-thinking and general stress. Transportation, hospital, and drug costs are frequently discussed as components of a woman's decision to seek health services, but do not seem to prevent women from seeking care in Cross River State.

\section{"If a woman is not taken care of, she will be thinking and this thinking will lead her to have high blood pressure. If she is made happy, her blood pressure will pump fine."}

- Female FGD participant, 2018

Women understand that eclampsia can cause maternal or fetal death and that women must register for ANC to avoid these outcomes. Convulsion is frequently associated with high BP, but little is known or discussed about the specific warning signs of hypertension or PE/E. Supportive community attitudes and greater awareness of women's pregnancy-related health needs are likely 
attributable to the emphasis on community engagement in Cross River State through a simultaneous study on potential impact women's groups can have on uptake of ANC services. Further analysis of these nuances is found in the accompanying women's group brief "Engaging Community Women's Groups for Improved Uptake of Antenatal Care Services in Cross River State, Nigeria," which summarizes learnings from that research.

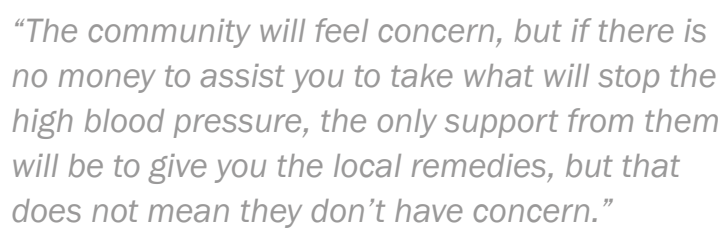

- Male FGD participant, 2018

\section{SURVIVOR'S EXPERIENCES}

Interviews with survivors document their care-seeking pathways, including their $\mathrm{PE} / \mathrm{E}$ experience, availability, and accessibility of essential services and commodities, and the outcomes of the pregnancy for women and their babies. Survivors' experiences can inform strategies to work more closely with communities and health facilities to improve access to, and use of, quality care.

Many survivors in Cross River State began attending ANC because they were aware of its importance, rather than any $\mathrm{PE} / \mathrm{E}$-related symptoms. These findings are encouraging and may be linked to the study on potential impact women's groups can have on uptake of ANC services. Further analysis of these nuances is found in the accompanying women's group brief "Engaging Community Women's Groups for Improved Uptake of Antenatal Care Services in Cross River State, Nigeria." However, some women continue to register late for ANC, after the first trimester in pregnancy.

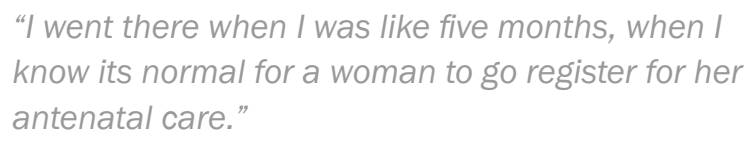

- Pre-eclampsia survivor, 2018

Women report that their $\mathrm{BP}$ and urine were routinely checked during ANC, but that the implications of high BP or proteinuria were not often discussed until the woman was pre-eclamptic and needing a caesarean section (CS).

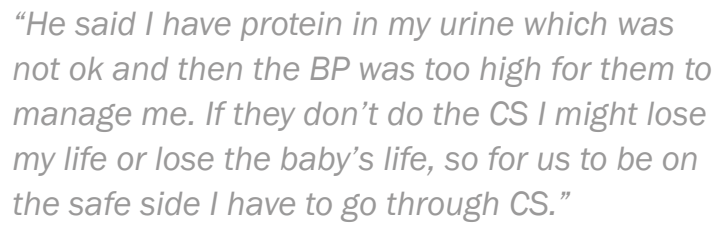

- Pre-eclampsia survivor, 2018

\section{DISCUSSION}

There is consistent and strong support from policy makers to scale up task-shifting of $\mathrm{MgSO}_{4}$ loading dose administration and referral to $\mathrm{PHC}$ providers. This favorable policy environment is substantiated by our evaluation of PHC workers' proven capacity to detect, treat, and manage hypertension and PE/E through administration of $\mathrm{MgSO}_{4}$ loading doses and referrals.

Facility capacity to detect, treat, and manage PE/E has improved, though variable and shifting funding streams impact $\mathrm{PE} / \mathrm{E}$ supply chains and procurement procedures. Inconsistent commodity supply impacts the sustainability of $P E / E$ detection and treatment, as well as the technical quality of ANC, maternity and PNC in facilities.

In Cross River, engagement with community groups led to improvements in women's use of health services by increasing their ability to actively engage with the health sector. In particular, women leaders are an important resource for providing health education to community women and play a significant role in bridging the gap between traditional beliefs and evidence-based practices.

One limitation of the data is the unequal sample sizes from ANC observations at baseline and endline. However, triangulating across different data sources and types renders our study findings robust.

\section{RECOMMENDATIONS}

- Streamline state procurement and enhance linkages to central distribution system;

- Continue mentoring and supportive supervision for PHC providers to detect and manage hypertension in pregnancy, including $\mathrm{PE} / \mathrm{E}$;

- Train providers, particularly at secondary facilities, on respectful maternity care to improve patientprovider interactions and experiential quality of care; and

- Sustain support to local women's groups to continue to improve community awareness, encourage early ANC, and improve health seeking behavior for pregnancy-related complications.

\section{REFERENCES}

1. DHS Nigeria, 2013.

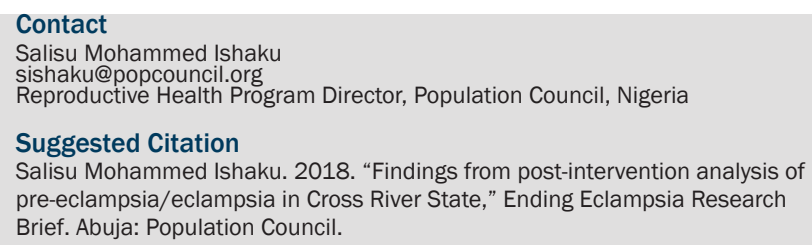

